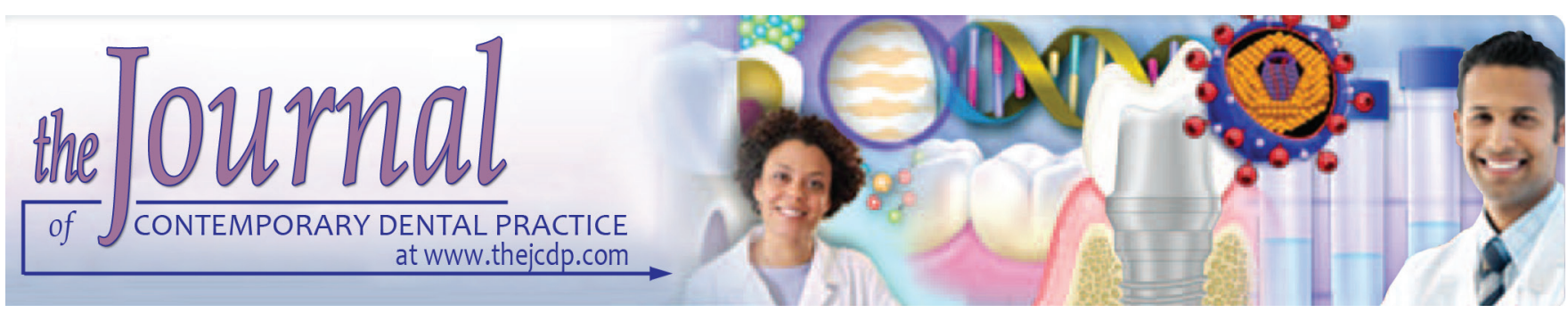

\title{
Vertebral Malalignment among Male Dentists with Work- related Musculoskeletal Pain in the United Arab Emirates
}

\author{
${ }^{1}$ Natheer H Al-Rawi, ${ }^{2}$ Hibba Yousef, ${ }^{3}$ Muna Khamis, ${ }^{4}$ Ouiza Belkadi, ${ }^{5}$ Shaikha Ahmed, ${ }^{6}$ Shaikha Ali
}

\begin{abstract}
Aim: Musculoskeletal pain (MSP) is a highly prevalent and debilitating disorder among dentists, often leading to early retirement. Poor ergonomics in the dental practice not only causes MSP, but can also lead to postural deviations as a result of an imbalance in muscle function. The aim of this study was to observe the relationship between MSP and vertebral deviations among male dentists in the United Arab Emirates (UAE).

Materials and methods: A total of 60 male dentists participated in this cross-sectional study. Standardized Nordic questionnaire (SNQ) was used to record MSP and PostureScreen Mobile ${ }^{\circledR}$ (PSM) application was used to observe postural deviations in the vertebral regions, including the neck, shoulders, upper back, lower back, and hips.
\end{abstract}

Results: About $83 \%$ of participants were suffering from MSP, with the lower back as the most commonly reported region (29\%). The pain characteristics reported were chronicity $(63 \%)$ and dullness (72\%). As for postural deviations, the most common site recorded was the neck region (72\%). The correlation between the regions of deviation and the regions of pain showed that the shoulder region was more significant than other regions.

Conclusion: Musculoskeletal pain and vertebral malalignment are common among practicing dentists, probably because of bad postural habits and lack of awareness. Preventive strategies to minimize the risk of developing these health problems are highly recommended.

Clinical significance: Work-related MSP (WRMSP) is a significant health problem among dentists and may be linked to the later development of vertebral deviations. Posture screen analysis is a quick, yet objective postural and movement screening tool in which findings can quickly be rendered to prospective dentists, so that custom postural and functional exercises may be correctly practiced.

\footnotetext{
${ }^{1-6}$ Department of Oral and Craniofacial Health Sciences, College of Dental Medicine, University of Sharjah, Sharjah, United Arab Emirates

Corresponding Author: Natheer $\mathrm{H}$ Al-Rawi, Department of Oral and Craniofacial Health Sciences, College of Dental Medicine, University of Sharjah, Sharjah, United Arab Emirates e-mail: nhabdulla@sharjah.ac.ae
}

Keywords: Dentists, Musculoskeletal pain, Posture screen, Vertebral malalignment.

How to cite this article: Al-Rawi $\mathrm{NH}$, Yousef $\mathrm{H}$, Khamis $\mathrm{M}$, Belkadi O, Ahmed S, Ali S. Vertebral Malalignment among Male Dentists with Work-related Musculoskeletal Pain in the United Arab Emirates. J Contemp Dent Pract 2018;19(7):773-777.

Source of support: Nil

Conflict of interest: None

\section{INTRODUCTION}

Dentistry is one of the most physically challenging careers, with musculoskeletal disorders becoming increasingly common worldwide. Musculoskeletal pain ranges between 64 and 93\%; lower back pain is more frequently experienced by dentists aged between 30 and 40 years. $^{1}$

Although dentists may be aware of proper work ergonomics, the complexity, psychomotor skills, and manual dexterity that are required often relegate appropriate work posture to the back of the operator's mind. A variety of factors contribute to the presence of WRMSP among dentists, including postural habits. Sitting in an awkward position increases stress on the posterior ligamentous structures of the spine, causing the muscles to adapt by lengthening or shortening, thereby weakening the spinal support and increasing the chance of injury. ${ }^{2}$ Over time, muscle imbalance may lead to structural damage and pain.

Muscle imbalance explains why most dentists with poor working posture experience MSP. For example, if dentists adopt a forward head posture (FHP) which places a high static load on the spine, they might experience neck pain. ${ }^{3}$ Prolonged static postures and poor positioning can cause increased anterior compression of the intervertebral discs and increased spinal cord pressure in the lumbar region. Eventually, this will put vertebral discs at risk of herniation and affect vital nerves in the surrounding area. ${ }^{2}$ 
Furthermore, improper ergonomics may cause the spine to curve more than normal or be misaligned, resulting in spine curvature disorders. This malalignment may manifest itself as scoliosis, which is an abnormal curvature of the spine to one side of the body; ${ }^{4}$ kyphosis, which is an excessive posterior curvature of the thoracic spine; ${ }^{5}$ or lordosis, which is an exaggerated curve of the lumbar spine. ${ }^{6}$ Furthermore, bad posture causes the head and shoulders to come forward, leading to FHP and rounded shoulders. ${ }^{7}$ Although various studies have been carried out to determine the postural risk factors experienced by dentists, the authors are aware of only a few studies that discuss the possible relationship between MSP and posture; this study aimed to find the relation between MSP and vertebral deviations among male dentists in the UAE.

\section{MATERIALS AND METHODS}

A cross-sectional study involving practicing male dentists across the cities of Abu Dhabi, Dubai, Sharjah, and Ajman. A total of 60 male dentists with more than 5 years of clinical experience were chosen to participate in the study using the convenient sampling method. The study consisted of two parts: the first part was answering the SNQ for the assessment of MSP, and the second part was posture evaluation, which was carried out using the PSM application (Posture Co Inc., Trinity, Florida, USA). The study was approved by the University of Sharjah Institutional Review Board (IRB-UOS), and all participants signed a written informed consent before enrollment in the study. Before each assessment, a room was set up in a standardized way to ensure uniform conditions throughout the test. According to Boland et al, a horizontal mark was placed on the floor to indicate where the participant standing position for the anterior photograph. Another vertical mark was placed perpendicular to the first to indicate positioning for the lateral photograph. The tablet (Apple iPad Air2) was placed on a stand exactly $3 \mathrm{~m}$ from the participant, with markers at a height of $1.5 \mathrm{~m}$ to standardize the image angle.

Dentists with fewer than 5 years of clinical experience, or with a history of nonwork-related musculoskeletal injuries, were excluded from the study. The SNQ was filled out by the participants after the procedure had been explained. The participants were instructed to stand at the cross marks in the upright position with their weight evenly distributed on both feet and to look at a marker placed at eye level on the wall in front of them. Two photographs were then taken of each participant, one from the left lateral view and the other from the back view (Fig. 1). With regard to MSP, only the regions of the neck, shoulders, upper back, lower back, and hips were considered; all other regions were excluded from the study.

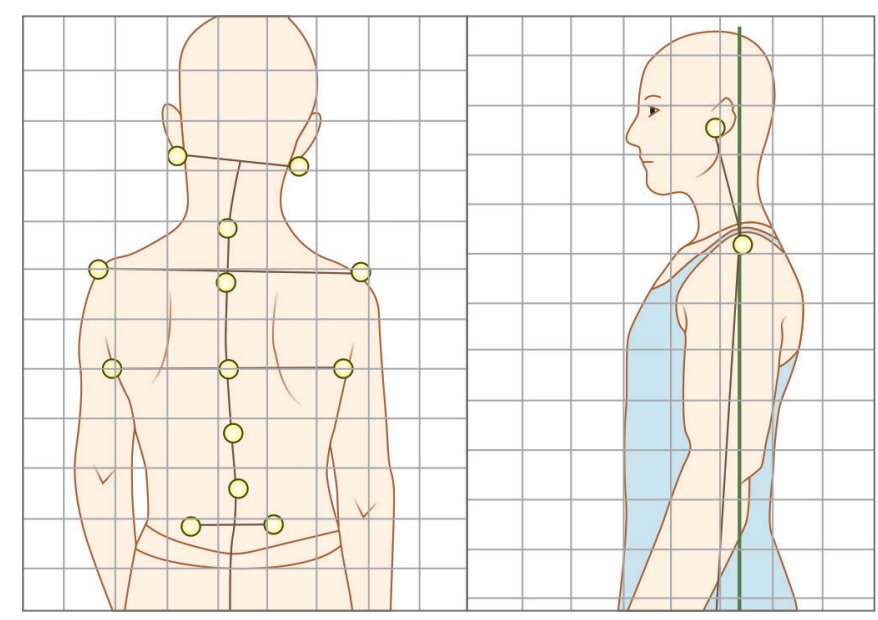

Fig. 1: Reference points on back and lateral view

Images captured with participants wearing minimal clothing were then analyzed by a physiotherapist using the PSM application. This application is a quick, objective postural screening tool, routinely used by professionals to determine and screen postural abnormalities. Analysis of the lateral view works by placing four points at different anatomic structures: the external auditory meatus, the cervical thoracic junction level of the acromioclavicular (AC) joint, the hip, and the knee. From the back view, the points applied were right and left of the inferior earlobes, level of C7 to T1, right and left of the AC joints, level of T4; right and left of T8, level of T12, level of L3, and right and left of the posterior superior iliac spine.

The PSM then calculated the following 10 quantitative data points using proprietary algorithms: head shift (lateral and longitudinal), head tilt, shoulder shift (lateral and longitudinal), shoulder tilt, hip shift (lateral and longitudinal), and hip tilt as interpreted by a physiotherapist. Statistical analysis was done using the Statistical Package for the Social Sciences version 21; values of less than 0.05 were considered statistically significant.

\section{RESULTS}

Of the 60 male dentists participating in the study, 28 (46\%) were general practitioners and $32(54 \%)$ were specialists. About $72 \%$ of the study sample had more than 11 years of experience. About $83 \%$ of the participants had reported MSP in the past year. The highest incidence was in the lower back region (29\%), followed by the neck (26\%), shoulders (25\%), hips (11\%), and upper back region (9\%) (Graph 1); 63\% of the participants experienced chronic pain, $18 \%$ experienced acute pain, and $18 \%$ did not specify the duration of pain. Dull pain was experienced by $72 \%$ of participants, $19 \%$ experienced sharp pain, and only $9 \%$ described the pain as throbbing pain. Regarding the deviations observed, the highest incidence was for the neck region $(72 \%)$, followed by the hip region (42\%), upper 


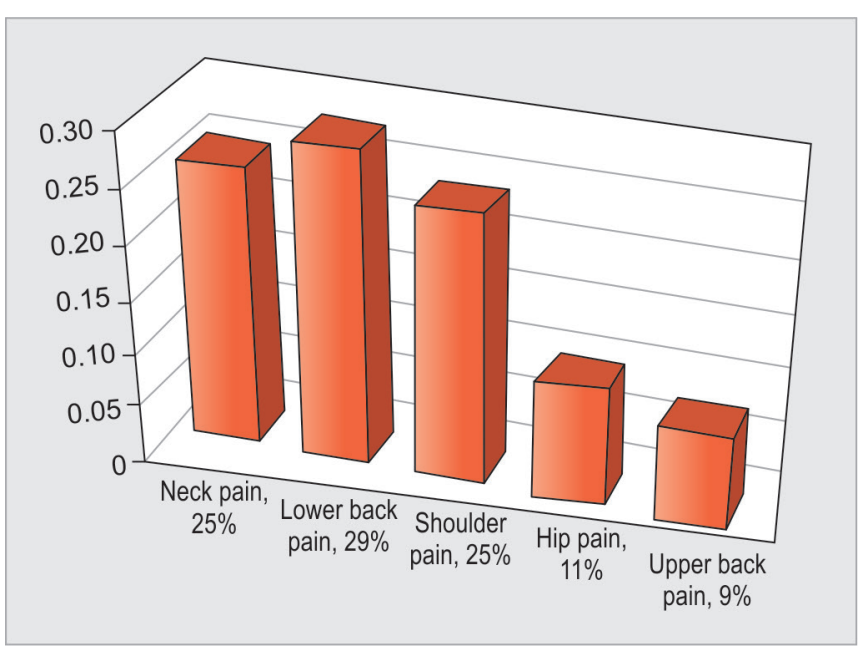

Graph 1: Regions of MSP

back $(38 \%)$, lower back $(17 \%)$, and lastly the shoulder region $(17 \%)$. Regarding dentists with neck deviations, $65 \%$ of them displayed a forward neck deviation, while another 7\% had a flat neck (Graph 2A). All 17\% of dentists with shoulder deviations displayed rounded shoulders (Graph 2B). Of the $38 \%$ of dentists with an upper back deviation, $20 \%$ had scoliosis, with the remaining $18 \%$ suffering from kyphosis (Graph 3A). Of the 17\% of dentists with lower back deviations, 9\% suffered from scoliosis and $8 \%$ had lordosis (Graph 3 B). Regarding hip deviations, $35 \%$ displayed an anterior pelvic tilt (APT), whereas $7 \%$ had only a posterior pelvic tilt (PPT) (Graph 4). The relationship between regions of pain and regions of deviation was analyzed using the Pearson chi-squared test, with the region of the shoulders showing a positive correlation (Table 1). Risk estimate revealed that shoulder deviation and hip deviation increased the odds of MSP by 1.976 and 1.609 respectively (Tables 2 and 3).

\section{DISCUSSION}

According to the World Health Organization, ${ }^{9}$ a musculoskeletal disorder is defined as a disorder of the muscles, tendons, peripheral nerves, or vascular system not directly resulting from an acute or instantaneous
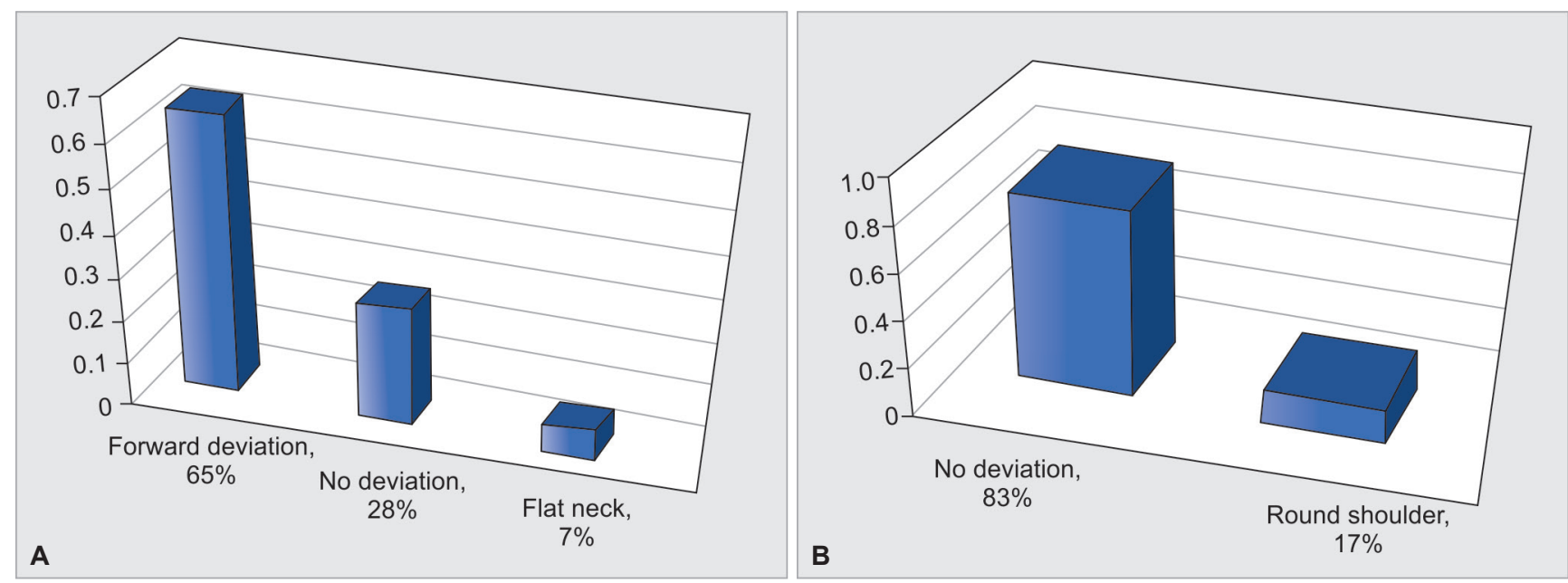

Graphs 2A and B: (A) Types of neck deviation, (B) Shoulder deviations
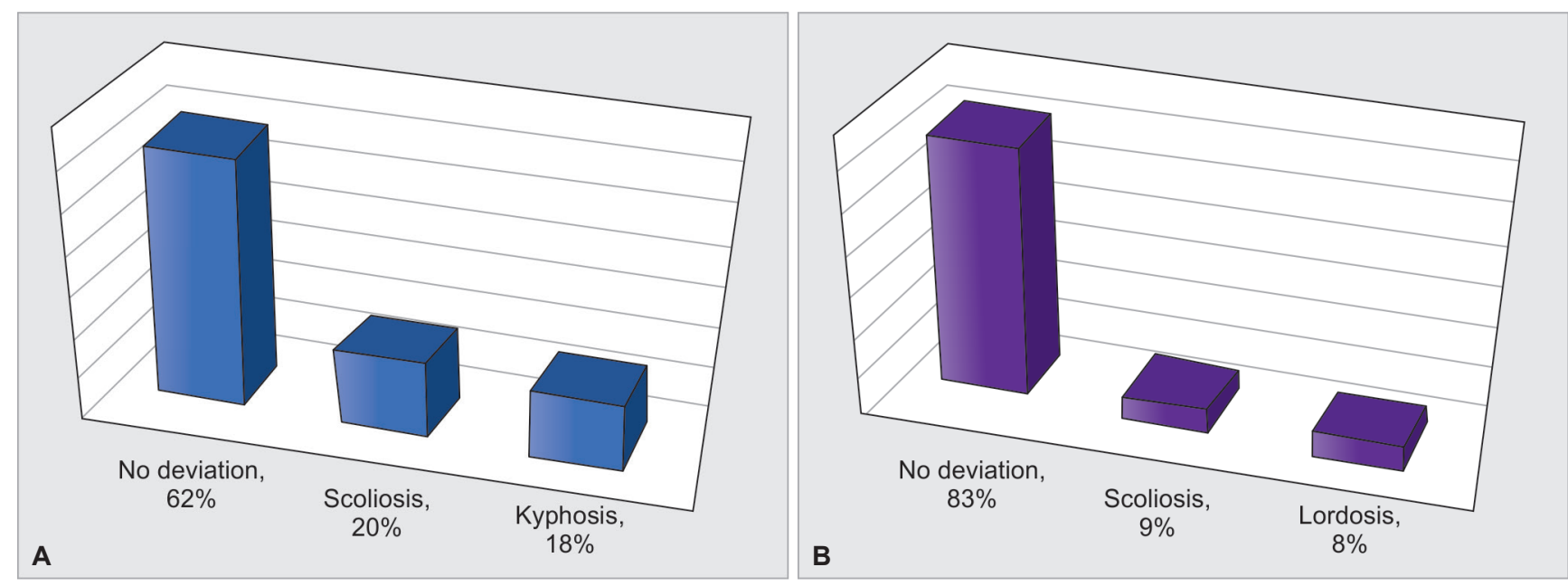

Graphs 3A and B: (A) Types of upper back deviation, (B) Lower back deviation 


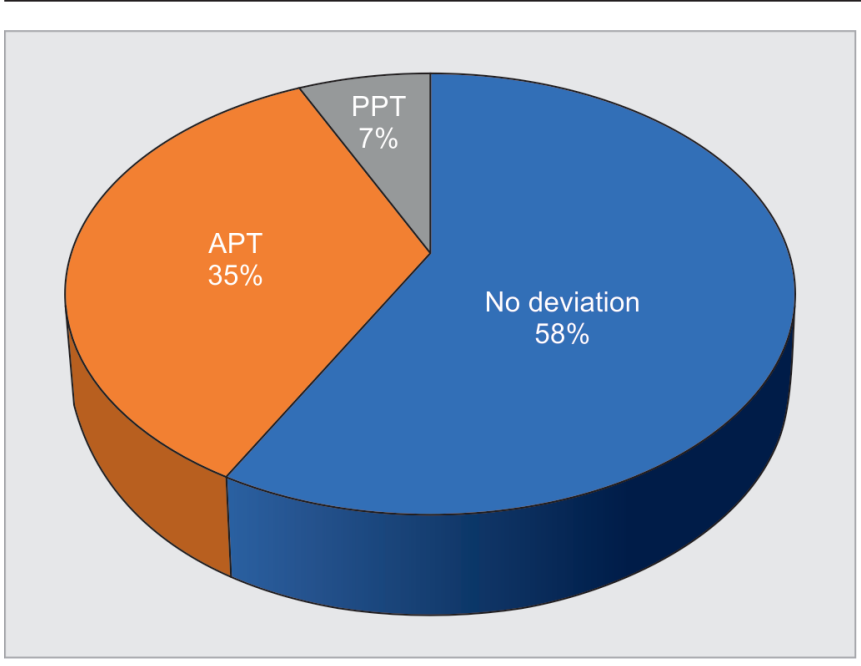

Graph 4: Types of hip deviations

Table 1: The relation between the region of pain and region of deviation

\begin{tabular}{lll}
\hline Region & Pearson chi-square & $p$-value \\
\hline Neck & 52.13 & 0.21 \\
Shoulders & 32.6 & $0.05^{*}$ \\
Upper back & 55.38 & 0.11 \\
Lower back & 54.7 & 0.19 \\
Hips & 63.53 & 0.46 \\
\hline
\end{tabular}

*Significant at $p \leq 0.05$

Table 2: Risk estimates of MSP in relation to shoulder deviation

\begin{tabular}{llll}
\hline & & \multicolumn{2}{c}{ 95\% confidence interval } \\
\cline { 3 - 4 } Region & Value & Lower & Upper \\
\hline $\begin{array}{l}\text { Odds ratio for WRMSP } \\
\text { (Yes/No) }\end{array}$ & 1.976 & 0.221 & 17.623 \\
$\begin{array}{l}\text { For cohort shoulder } \\
\text { deviation = Yes }\end{array}$ & 1.800 & 0.256 & 12.667 \\
$\begin{array}{l}\text { For cohort shoulder } \\
\text { deviation = No }\end{array}$ & 0.911 & 0.714 & 1.163 \\
\begin{tabular}{l} 
No. of valid cases \\
\hline
\end{tabular} & 60 & & \\
\hline
\end{tabular}

Table 3: Risk estimates of MSP in relation to hip deviation

\begin{tabular}{lllc}
\hline & & \multicolumn{2}{c}{$95 \%$ confidence interval } \\
\cline { 3 - 4 } Region & Value & Lower & Upper \\
\hline $\begin{array}{l}\text { Odds ratio for WRMSP } \\
\text { (Yes/No) }\end{array}$ & 1.609 & 0.371 & 6.982 \\
$\begin{array}{l}\text { For cohort shoulder } \\
\text { deviation = Yes }\end{array}$ & 1.361 & 0.498 & 3.717 \\
$\begin{array}{l}\text { For cohort shoulder } \\
\text { deviation = No }\end{array}$ & 0.845 & 0.530 & 1.350 \\
\begin{tabular}{l} 
No. of valid cases \\
\hline
\end{tabular} & 59 & & \\
\hline
\end{tabular}

event. This disorder has serious consequences for both the lifestyle and career of a dentist, as it has proven to be the leading cause of early retirement among dental practitioners. ${ }^{10}$

The findings of this study indicate that dentists are likely to suffer from MSP and that the pain is usually characterized by chronicity and dullness. In the present study, around $51 \%$ of dentists reported MSP in more than one region, and $30 \%$ of dentists reported MSP in only one region. This is in accordance with another study conducted in the $\mathrm{UAE}^{11}{ }^{11}$ which found that $67 \%$ of dentists across the Emirates suffer from MSP, with chronic, dull pain being the most common. Furthermore, both the present and the earlier study found that the most common regions of pain were the lower back and neck region. If the neck is flexed forward and rotated for long periods of time, this creates muscle tension in the neck, back, and shoulder regions. In the present study, different deviations were observed in the neck, shoulders, upper back, lower back, and hip regions. The FHP is one of the most common contributing conditions to neck pain and was reported in about $65 \%$ of dentists in the present study. It is characterized by the forward positioning of the head away from the centerline of the body, or forward neck bending, which places the head in front of the shoulders. This bending position exerts high pressure on the surrounding structures, including the muscles and joints of the neck. ${ }^{7}$ The FHPs are common among dentists because they hold the neck and head in an unbalanced forward position to gain better visibility during dental procedures. This can result in a pain pattern that is often referred to as "tension neck syndrome." Flat neck, also known as straight neck, is another bony deformity of the cervical spine region. In individuals with a flat neck, the cervical spine loses its usual C-shape curvature to become flat. ${ }^{7}$ The flat neck was reported in about $6 \%$ of the sample of the present study.

Another common abnormal body posture is the rounded shoulders posture. Rounded shoulders are defined as the rolling forward of the shoulders causing a stooped posture. Rounded shoulders can lead over time to other postural deviations, such as the FHP previously mentioned or kyphosis. ${ }^{7}$ Seven dentists with forwarding neck deviation reported rounded shoulder and nine of the participating dentists with forwarding neck deviation reported kyphosis.

Kyphosis is a condition of spinal deformity in which the vertebrae change from a cylindrical to a wedge shape. With it, the spine begins to curve forward, giving the upper back a rounded appearance. A kyphotic posture refers to the excessive posterior curvature of the thoracic spine. ${ }^{5}$ Another common spinal deformity is scoliosis, which causes an abnormal curvature of the spine to one side of the body. Scoliosis tends to develop gradually, creating either C-or S-shaped curvatures when the spine is viewed from the back. ${ }^{4}$ Lordosis is an increased or exaggerated curve of the lumbar spine affecting normal mobility. It mostly affects the lumbar spine, but can also be found in the cervical spine. ${ }^{6}$ One abnormal postural condition of the hip is known as the PPT, which is a 
backward rotation of the pelvis that brings the pubic symphysis (anterior) of the pelvis upward and the sacrum (posterior) downward. Opposite to the PPT is the APT, a more common hip condition in which the pelvis tilts forward. $^{12}$

Deviations in posture can result from muscle imbalances. Muscles work in opposing groups, known as the tonic and phasic muscles. Tonic muscles tend to tighten, whereas phasic muscles tend to relax. Maintaining an abnormal position for prolonged periods of time can cause an imbalance of these two groups of muscles, causing a deviation around the joint to which they attach. This ultimately leads to deviations in posture among dentists because of the awkward positions they maintain throughout their daily routine. ${ }^{13}$ Improper ergonomics of both the placement of the patient and equipment add to the postural stress placed on the dentist. Sitting on chairs that are too low or too high with inadequate lower back support combined with positioning patients too high leads to stress and strain in the neck, shoulder, and lower back region. ${ }^{3}$ Since MSP has substantial effects on a dentist's health and lowers their productivity because of missed appointments, management and preventive strategies should be implemented to minimize MSP as much as possible. Prevention starts with maintaining a healthy lifestyle by performing aerobic and strengthening exercises, preferably 3 to 4 times per week. ${ }^{3}$ In the clinic, positioning the operator's chair correctly and making sure that it has good lumbar support has shown to be effective in preventing MSP. Practicing proper techniques at work with a trained assistant ${ }^{14}$ and keeping the most frequently used equipment $50 \mathrm{~cm}$ from the operator are also recommended. ${ }^{3}$ Moreover, studies have shown that the use of magnification is associated with decreased neck and lower back pain as it allows the dentist to maintain a proper posture. ${ }^{15}$ To increase the blood flow to the muscles and recharge for the next appointment, dentists are advised to take short breaks between appointments and stretch their back, neck, and arms. ${ }^{16}$ Lastly, dentists should make every effort to develop preventive strategies and have their posture and spine evaluated regularly in order to maximize their work efficiency and ensure a long and successful clinical career. ${ }^{1}$ The small sample size and the one gender selection is one of the limitations of this study.

\section{CONCLUSION}

Work-related MSP is a significant health problem among dentists and may be linked to the later development of vertebral deviations. Dentists should be aware of correct posture and should be encouraged to take regular rest intervals between patients. By minimizing the identified risk factors through following ergonomic tips, the occurrence of MSP and vertebral deviations can be prevented or decreased.

\section{ACKNOWLEDGMENT}

The authors would like to thank Miss Lina Elsayed, the physiotherapist from the College of Health Sciences, University of Sharjah for her help in identifying the points used in PSM application.

\section{REFERENCES}

1. Abbas SB, Qazi SR, Iftekhar S, Iqbal MU. Musculoskeletal disorders among dentists and dental students. Pak Oral Dent J 2015 Sep;35(3):461-465.

2. Ayatollahi J, Ayatollahi F, Ardekani AM, Bahrololoomi R, Ayatollahi J, Ayatollahi A, Owlia MB. Occupational hazards to dental staff. Dent Res J (Isfahan) 2012 Jan;9(1):2-7.

3. Pîrvu C, Pătraşcu I, Pîrvu D, Ionescu C. The dentist's operating posture-ergonomic aspects. J Med Life 2014 Jun;7(2):177-182.

4. Janicki JA, Alman B. Scoliosis: review of diagnosis and treatment. Pediatr Child Health 2007 Nov;12(9):771-776.

5. Kisner, C.; Colby, LA. Therapeutic exercise: foundations and techniques. 5th ed. Philadelphia (PA): F.A. Davis; 2007.

6. Been E, Kalichman L. Lumbar lordosis. Spine J 2014 Jan;14(1):87-97.

7. Kim EK, Kim JS. Correlation between rounded shoulder posture, neck disability indices, and degree of forward head posture. J Phys Ther Sci 2016 Oct;28(10):2929-2932.

8. Boland DM, Neufeld EV, Ruddell J, Dolezal BA, Cooper CB. Inter- and intra-rater agreement of static posture analysis using a mobile application. J Phys Ther Sci 2016 Dec;28(12): 3398-3402.

9. WHO. Identification and control of work-related diseases: report of a WHO Expert committee. WHO Tech Rep Ser 1985;174:7-11.

10. Rehman K, Ayaz H, Urooj W, Shah R. Work-related musculoskeletal disorders among dental practitioners in Khyber Pakhtunkhwa. Pak Oral Dent J 2013 Dec;33(3):531-534.

11. Al-Rawi NH, El Khatib H, Rajoub L, El-Sayed M, Naji R, Youssef R, Al Kawas S. Work-related musculoskeletal pain among different dental specialists in United Arab Emirates. J Contemp Dent Pract 2016 Aug;17(8):639-644.

12. Levangie, PK.; Norkin, CC. Joint structure and function: a comprehensive analysis. 4 th ed. Philadelphia (PA): F.A. Davis Co; 2005.

13. Hardy J. Pain management: a practical guide for clinicians. J R Soc Med 2002 Sep;95(9):470-471.

14. Prudhvi K, Murthy K. Self-reported musculoskeletal pain among dentists in Visakhapatnam: a 12-month prevalence study. Indian J Dent Res 2016 Jul-Aug;27(4):348-352.

15. Dajpratham P,Ploypetch T, KiattavorncharoenS, Boonsiriseth $K$. Prevalence and associated factors of musculoskeletal pain among the dental personnel in a dental school. J Med Assoc Thai 2010 Jun;93(6):714-721.

16. Gosavi SS, Gosavi SY, Jawade RS. Posturedontics: reducing the stress in dentistry. World J Dent 2012 Oct;3(4):335-339. 